\title{
Shape-constrained Symbolic Regression - Improving Extrapolation with Prior Knowledge
}

G. Kronberger

Josef Ressel Center for Symbolic Regression, University of Applied Sciences Upper Austria, Softwarepark 11, 4232 Hagenberg, Austria

F. O. de Franca

folivetti@ufabc.edu.br Center for Mathematics, Computation and Cognition (CMCC), Heuristics, Analysis and Learning Laboratory (HAL), Federal University of ABC, Santo Andre, Brazil

B. Burlacu and C. Haider and M. Kommenda Josef Ressel Center for Symbolic Regression, University of Applied Sciences Upper Austria, Softwarepark 11, 4232 Hagenberg, Austria

\begin{abstract}
We investigate the addition of constraints on the function image and its derivatives for the incorporation of prior knowledge in symbolic regression. The approach is called shape-constrained symbolic regression and allows us to enforce e.g. monotonicity of the function over selected inputs. The aim is to find models which conform to expected behaviour and which have improved extrapolation capabilities. We demonstrate the feasibility of the idea and propose and compare two evolutionary algorithms for shapeconstrained symbolic regression: i) an extension of tree-based genetic programming which discards infeasible solutions in the selection step, and ii) a two population evolutionary algorithm that separates the feasible from the infeasible solutions. In both algorithms we use interval arithmetic to approximate bounds for models and their partial derivatives. The algorithms are tested on a set of 19 synthetic and four real-world regression problems. Both algorithms are able to identify models which conform to shape constraints which is not the case for the unmodified symbolic regression algorithms. However, the predictive accuracy of models with constraints is worse on the training set and the test set. Shape-constrained polynomial regression produces the best results for the test set but also significantly larger models 1
\end{abstract}

Keywords

Symbolic regression, Genetic programming, Shape-constrained regression

\section{Introduction and Motivation}

Dynamical systems and processes in critical application areas such as control engineering require trustworthy, robust and reliable models that strictly conform to behavioral expectations according to domain-specific knowledge, as well as safety and performance criteria. However, such models cannot always be derived from first principles and theoretical considerations alone. Thus, they must be determined empirically. From this perspective, models used in system identification can be categorized as:

\footnotetext{
${ }^{1}$ This work has been accepted to be published by Evolutionary Computation, MIT Press
} 
- White-box. Derived from first principles, explicitly include domain knowledge, can be valid for a wide range of inputs even beyond available observations and allow to make far-reaching predictions (extrapolation).

- Gray-box. Derived from data, with known internals, open for further inspection. Can be validated against domain knowledge. Only valid for inputs which are similar to observations that are used for model fitting.

- Black-box. Derived from data, with unknown internals (e.g., neural networks), difficult or impossible to inspect and/or validate.

In-between the two extremes there is a whole spectrum of gray-box models which combine aspects of both extremes (Ljung, 2010). The proponents of "explainable AI" propose to apply models on the white end of this spectrum either directly (Rudin, 2019) or as an approximation of black-box models in AI technologies.

Empirical, data-driven models are required in scenarios where first principles models are infeasible due to engineering or financial considerations. Ensuring that empirical models conform to expected system behavior, correctly reflect physical principles, and can extrapolate well on unseen data remains an open challenge in this area.

Symbolic regression (SR) is especially interesting in this context because SR models are closed-form expressions which are structurally similar to white-box models derived from first principles. The aim in SR is to identify the best function form and parameters for a given data set using common mathematical operators and functions which serve as building blocks for the function form (Koza, 1992). This is in contrast to other forms of regression analysis where the function form is pre-specified and only the numerical coefficients are optimized through model fitting.

It should be noted however that SR is fundamentally a purely data-driven approach. It does not require prior information about the modelled process or system and leads to gray-box models which can be hard to understand. SR algorithms therefore favor shorter expressions and use simplification to facilitate detailed analysis of their models.

The most well-known solution methods for SR includes tree-based genetic programming (GP) variants (Koza, 1992), linear GP (Oltean and Grosan, 2003) including Pareto-GP (Smits and Kotanchek, 2005), Cartesian GP (Miller and Harding, 2008), grammatical evolution (GE) (O'Neill and Ryan, 2001), and gene expression programming (GEP) (Ferreira, 2001). More recent developments of SR include Geometric Semantic GP (Moraglio et al., 2012; Pawlak and Krawiec, 2018; Ruberto et al., 2020), Multipleregression GP (Arnaldo et al., 2014), and a memetic evolutionary algorithm using a novel fractional representation for SR (Sun and Moscato, 2019). There are also several nonevolutionary algorithms for SR including fast function extraction (FFX) (McConaghy, 2011), SymTree (de França, 2018), prioritized grammar enumeration (PGE) (Worm and Chiu 2013). Recently several neural network architectures have been used for symbolic regression (Sahoo et al., 2018, Kim et al., 2019, Petersen et al., 2019, Udrescu and Tegmark, 2020).

This contribution introduces an extension of SR, which allows to include vague prior knowledge via constraints on the shape of SR model image and derivatives. We therefore call this approach shape-constrained SR as it is a specific form of shape-constrained regression. The main motivation is, that even in data-based modelling tasks, partial knowledge about the modelled system or process is often available, and can be helpful to produce better models which conform to expected behaviour and might improve extrapolation. Such information could be used for: 
1. data augmentation to decrease the effects of noisy measurements or for detecting implausible outliers

2. fitting the model in input space regions where observations are scarce or unavailable

3. distinguishing between correlations in observation data and causal dependencies

4. increasing the efficiency of the statistical modelling technique.

\subsection{Problem Statement}

We focus on the integration of prior knowledge in SR solvers in order to find expressions compatible with the expected behavior of the system.

Our assumptions are that we have data in the form of measurements as well as side information about the general behaviour of the modelled system or process that however is insufficient to formulate an explicit closed-form expression for the model. We additionally assume that the information can be expressed in the form of constraints on the function output as well as its partial derivatives.

The task is therefore to find a closed-form expression which fits the data, possibly with a small error, and conforms to our expectation of the general behaviour of the system. In particular, the model must not violate shape constraints when interpolating and extrapolating within a bounded input space.

We investigate the hypotheses that (i) interval arithmetic can be used to approximate bounds for the image of SR models and their partial derivatives and therefore reject non-conforming models within SR solvers, and (ii) that the predictive accuracy of SR models can be improved by using side information in the form of shape constraints.

\section{Related Work}

The issue of knowledge integration into machine learning methods including genetic programming has been discussed before.

Closely related to this work is the work of Bladek and Krawiec (2019) who have discussed using formal constraints in combination with symbolic regression to include domain knowledge about the monotonicity, convexity or symmetry of functions. Their approach called Counterexample-Driven GP utilizes satisfiability modulo theories (SMT) solvers to check whether candidate SR models satisfy the constraints and to extend the training set with counter examples. Bladek and Krawiec (2019) observe that is very unlikely for GP to synthesize models which conform to constraints. The difference to this work is that we do not support symmetry constraints and instead of a SAT solver we use interval arithmetic to calculate bounds for the function image and its partial derivatives. We test our approach on a larger set of harder benchmark problems.

Versino et al. (2017) have specifically studied the generation of flow stress models for copper using GP with and without knowledge integration. They have investigated four different scenarios: i) a canonical GP solver that returns a Pareto front of model quality and complexity, ii) knowledge integration by means of transformed variables, samples weighting, introduction of artificial points, introduction of seed solutions, iii) search for the derivative of the expression and then reconstructing the original equation by integration, iv) search derivatives but with additional artificial data points.

This work emphasized the importance of integrating prior knowledge in the model fitting process. The authors observed that the canonical GP solver presented unpredictable behavior w.r.t. the physical constraints, thus the sampled data set alone was insufficient to guide the solver into a model with both low error and physical feasibility. 
In contrast to our approach which guarantees that solutions conform to prior knowledge, all techniques tried in (Versino et al., 2017) are optimistic and might produce infeasible solutions. Additionally, adding artificial data points is subjective and does not scale to high-dimensional problems.

Schmidt and Lipson (2009) studied the influence of integrating expert knowledge into the evolutionary search process through a process called seeding. First the authors generated approximation solutions by either solving a simpler problem or finding an approximate solution to a more complex problem. These solutions are then used during the seeding procedure by inserting approximations into the initial population, shuffled expressions and building blocks. The authors found that seeding significantly improves the convergence and fitness average performance when compared to no seeding. Among the seeding procedures, the seeding of building blocks was the most successful allowing faster convergence even in more complex problems. Again, seeding is an optimistic approach and does not guarantee that the final SR solution conforms to prior knowledge.

Stewart and Ermon (2017) investigated how to use prior knowledge in artificial neural networks. Specifically, they incorporate physical knowledge into motion detection and tracking and causal relationships in object detection.

Even though the experiments were all small scaled, they showed promising results and opened up the possibility of teaching an artificial neural network by only describing the properties of the approximation function. This idea is similar to the idea of shapeconstraints.

Recently an approach for knowledge integration for symbolic regression with a similar motivation to this work has been described in (Li et al., 2019). Instead of shape constraints the authors make use of semantic priors in the form of leading powers for symbolic expressions. They describe a Neural-Guided Monte Carlo Tree Search (MCTS) algorithm to search of SR models which conform to prior knowledge. The authors use a context-free grammar for generating symbolic expressions and use MCTS for generating solutions whereby a neural network predicts the next production rule given a sequence of already applied rules and the leading power constraints.

The algorithm was compared against variations of MCTS and a GP implementation using the DEAP framework (Fortin et al. 2012)

The results show a superior performance of neural-guided MCTS with a high success rate for easier instances but much lower rates on harder instances which were however still higher than the success rate of GP.

One aim of our work is improving extrapolation of SR solutions. Similarly to polynomial regression, SR models might produce extreme outputs values when extrapolating. Interestingly, extrapolation behaviour of SR models has been largely ignored in the literature with only a few exceptions.

Castillo et al. (2013) investigated the difference in the response variation of different models generated by GP to three different data sets. They have shown that in some situations a GP algorithm can generate models with different behavior while presenting a similar $R^{2}$ value. These results motivate the need of a post analysis of the validity of the model w.r.t. the system being studied and a verification of how well such models extrapolate.

The extrapolation capability of GP is also studied in (Castillo et al. 2003). In this work, the authors evaluate and compare the best solution obtained by a GP algorithm to a linear model which uses the transformed variables found by the GP model.

The experiments suggest that the GP models have good interpolation capabilities but with a moderate extrapolation error. The proposed approach presented good inter- 
polation and extrapolation capabilities, suggesting that at the very least, the GP models can guide the process of building a new transformed space of variables.

Another relevant result was obtained by Kurse et al. (2012) who used Eureqa (a commercial SR solver) to find analytical functions that correctly modelled complex neuromuscular systems. The experiments showed that the GP solver was capable of extrapolating the data set much better than the traditional polynomial regression, used to model such systems.

Stewart and Ermon (2017) trained a neural network by introducing prior knowledge through a penalty term of the loss function such that the generated model is consistent to a physics behavior. Different from our work, this generates a black box model of the data. Finally, Zhu et al. (2019) incorporate the appropriate partial differential equations into the loss functions of a neural network and, by doing so, they can train their model on unlabeled data, this approach requires more information than usually available when modelling studied observations.

\section{Methods}

In this section we describe in detail the methods proposed in this paper to integrate prior knowledge into SR solvers using interval arithmetic and shape constraints. In summary, we extend two GP variants to support shape-constrained SR: a tree-based GP approach with optional memetic improvement of models parameters and the InteractionTransformation Evolutionary Algorithm (ITEA) (de Franca and Aldeia, 0). The extended algorithms are compared against the original versions on a set of benchmark problems. For the comparison we calculate the number of constraint violations and the training as well as the test errors and qualitatively assess the extrapolation behaviour.

\subsection{Shape-constrained Regression}

Shape-constrained regression allows to fit a model whereby certain characteristics of the model can be constrained. It is a general concept, that encompasses a diverse set of methods including parametric and non-parametric, as well as uni-variate and multi-variate methods. Examples include isotonic regression (Wright et al., 1980, Tibshirani et al., 2011), monotonic lattice regression (Gupta et al., 2016), nonparametric shape-restricted regression (Guntuboyina et al., 2018), non-negative splines (Papp and Alizadeh, 2014), and shape-constrained polynomial regression (Hall, 2018).

In shape-constrained regression, the model is a function mapping real-valued inputs to a real-valued output and the constraints refer to the shape of the function. For instance if the function $f(\mathbf{x})$ should be monotonically increasing over a given input variable $x_{1}$ then we would introduce the monotonicity constraint

$$
\frac{\partial f}{\partial x_{1}}(\mathbf{x}) \geq 0, \mathbf{x} \in \mathcal{S} \subseteq \mathbb{R}^{d}
$$

Similarly, we could enforce concavity/convexity of a function by constraining second order derivatives. Usually the input space for the model is limited for example to a $d$-dimensional box $\mathcal{S}=\left[\inf _{1}, \sup _{1}\right] \times\left[\inf _{2}, \sup _{2}\right] \times \cdots \times\left[\inf _{d}, \sup _{d}\right] \subseteq \mathbb{R}^{d}$.

In general it is possible to include constraints for the model and its partial derivatives of any order. However, in practice first and second order partial derivatives are most relevant. The set of constraints $C$ contains expressions that are derived from the model via one of the operators $\mathrm{Op}$ and linearly transformed using a sign $s$ and threshold $c$. We use this representation of shape constraints to simplify checking of constraints. All 
constraint expressions $c_{i} \in C$ must not be positive for feasible solutions.

$$
C=\left\{\mathrm{s} \cdot \mathrm{Op}(f)(\mathbf{x})-c \leq 0 \mid \mathrm{Op} \in\left\{\operatorname{id}(f), \frac{\partial^{n} f}{\partial^{n} x_{1}}, \ldots \frac{\partial^{n} f}{\partial^{n} x_{d}}\right\}, c \in \mathbb{R}, \mathrm{s} \in\{1,-1\}, n>0\right\}
$$

It is important to mention that shape constraints limit the function outputs and partial derivatives, but the optimal function which fits the observed data still needs to be identified by the algorithm. Shape-constrained regression is a general concept which is applicable to different forms of regression analysis. For specific models like multivariate linear regression or tree-based methods (e.g., XGBoost), it is easy to integrate shape constraints. For other models, for instance, polynomial regression, it is harder to incorporate shape constraints (Hall, 2018; Ahmadi and Majumdar, 2019).

Generally, one can distinguish optimistic and pessimistic approaches to shapeconstrained regression (Gupta et al., 2016). Optimistic approaches check the constraints only for a finite set of points from the input space and accept that the identified model might violate the constraints for certain elements from the input space. Pessimistic approaches calculate bounds for the outputs of the model and its partial derivatives to guarantee that identified solutions are in fact feasible. However, pessimistic approaches might reject optimal models as a consequence of overly wide bounds. In this paper, we decided to study a pessimistic approach and calculate bounds for SR models and their derivatives using interval arithmetic.

\subsection{Interval Arithmetic}

Interval Arithmetic (IA) is a method for calculating output ranges for mathematical expressions (Hickey et al., 2001). It has many uses such as dealing with uncertainties stemming from inaccurate representations and verifying boundary conditions. An interval is represented as $[a, b]$ with $a \leq b$, named lower and upper endpoints, respectively.

If we have a function $f\left(x_{1}, x_{2}\right)=x_{1}+x_{2}$, for example, and knowing the intervals for each variable to be $x_{1} \in[a, b], x_{2} \in[c, d]$, we can say that the image of function $f$ will be in the interval $[a+c, b+d]$. IA defines the common mathematical operations and functions for such intervals. It is easy to see that IA can only give bounds for the expression instead of an accurate range because it does not track dependencies between arguments of operators. For instance, the IA result for the expression $x_{1}-x_{1}$ with $x_{1} \in[a, b]$ is the interval $[a-b, b-a]$ instead of $[0,0]$.

IA has previously been used for SR for improving model quality as it allows to detect and reject partially defined functions resulting e.g. from division by zero or taking the logarithm of a non-positive number. The use of IA for SR was first proposed in Keijzer, 2003) where IA was used to verify that a given expression is defined within the domain spanned by the variable values in the data set. The author showed that this indeed helps avoiding partially defined functions even when using standard division instead of the commonly used protected division operator.

Pennachin et al. (2010) used Affine Arithmetic, an extension to IA, for finding robust solutions. The authors observed that the models generated by their algorithm were robust w.r.t. extrapolation error.

In (Dick, 2017) the approach of (Keijzer, 2003) was extended by introducing crossover and mutation operators that make use of IA to generate feasible expressions. The experiments showed a faster convergence rate with interval-aware operators.

We also include IA into SR. Our work is in fact a generalization of the idea discussed in (Keijzer, 2003) and (Pennachin et al. , 2010), but instead of limiting the use of IA to detect only whether a function is partial or not, we also expand it to test for 
other properties such as monotonicity. Another difference is that we assume that the considered intervals are derived from prior knowledge, instead of being inferred from the data.

\subsection{Genetic Programming for Shape-constrained Symbolic Regression}

We integrate IA into two solvers for SR. In this section we describe the integration into tree-based GP (Koza, 1992) and in the next section we describe the integration into ITEA.

We use a tree-based GP variation with optional memetic local optimization of SR model parameters which has produced good results for a diverse set of regression benchmark problems (Kommenda et al., 2020).

The pseudo-code for our GP algorithm for shape-constrained symbolic regression is shown in Algorithm 1. Solution candidates are symbolic expressions encoded as expression trees. Parameters of the algorithm are the function set $\mathcal{F}$, the terminal set $\mathcal{T}$, the maximum length (number of nodes) $L_{\max }$ and maximum depth $D_{\max }$ of expression trees, the number of generations $G_{\max }$, the population size $N$, the mutation rate $m$, the tournament group size $p$, and the number of iterations for memetic parameter optimization $n_{\mathrm{Opt}}$. The algorithm uses tournament selection, generational replacement and elitism. New solution candidates are produced via sub-tree crossover and optional mutation. The initial population is generated with the PTC2 algorithm (Luke, 2000). All evolutionary operators respect the length and depth limits for trees.

Shape constraints are handled during fitness evaluation. First we calculate intervals for the function output as well as the necessary partial derivatives using the known intervals for each of the input variables and check the output intervals against the constraint bounds. If a solution candidate violates any of the shape constraints the solution candidate is assigned the worst possible fitness value. In a second step, the prediction error is calculated only for the remaining feasible solution candidates. The solution candidate with the smallest error within a group is selected as the winner in tournament selection.

We assume that the constraints are provided via a set $C$ as given in Section 3.1

\subsubsection{Fitness Evaluation}

Function Evaluate calculates the vector of residuals from the predictions for inputs $X$ and the observed target values $y$. We use the normalized mean of squared errors (NMSE) as the fitness indicator. NMSE is the mean of squared residuals scaled with the inverse variance of $y$. As described in (Keijzer, 2003) we implicitly scale all symbolic regression solutions to match the mean and variance of $y$. Therefore, we know that the worst possible prediction (i.e. a constant value) has an NMSE of one and we use this value for infeasible solution candidates.

\subsubsection{Optional Local Optimization}

Procedure Optimize locally improves the vector of numerical coefficients $\theta \in \mathbb{R}^{\text {dim }}$ of each model using non-linear least-squares fitting using the Levenberg-Marquardt algorithm. It has been demonstrated that gradient-based local improvement improves symbolic regression performance (Topchy and Punch, 2001, Kommenda et al., 2020). Here we want to investigate whether it can also be used in combination with shape constraints. To test the algorithms with and without local optimization we have included the number of local improvement iterations $n_{\mathrm{Opt}}$ as a parameter for the GP algorithm.

The local improvement operator extracts the initial parameter values $\theta_{\text {init }}$ from the solution candidates and updates the values after optimization. Therefore, improved parameter values $\theta^{\star}$ become part of the genome and can be inherited to new solution 

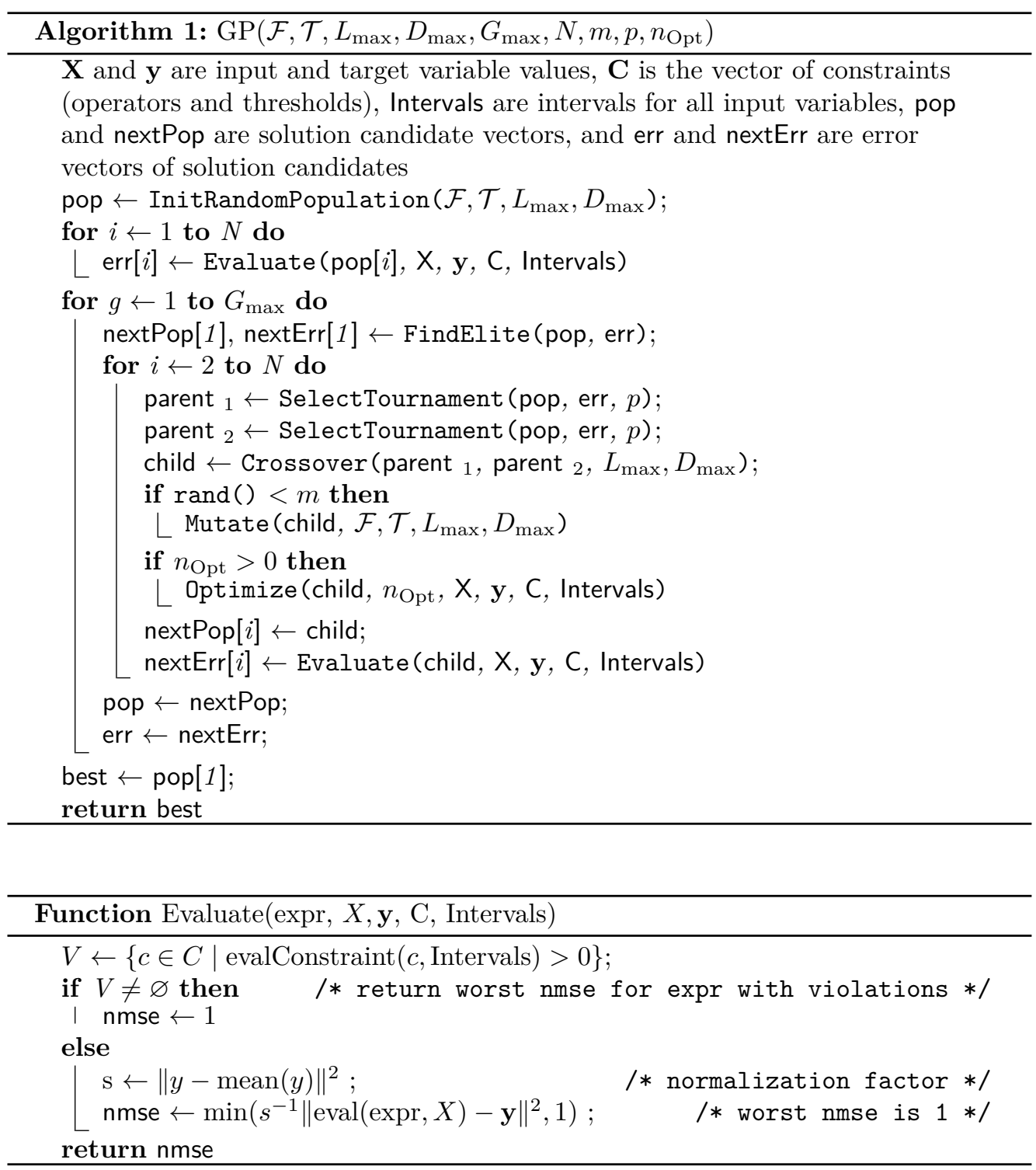

candidates (Lamarckian learning (Houck et al. 1997)).

\subsection{Interaction-Transformation Evolutionary Algorithm}

The second SR method that we have extended for shape-constrained SR is the Interaction-Transformation Evolutionary Algorithm (ITEA). ITEA is an evolutionary algorithm that relies on mutation to search for an optimal Interaction-Transformation expression (de França, 2018) that fits a provided data set. The Interaction-Transformation (IT) representation restricts the search space to simple expressions following a common pattern that greatly simplifies optimization of model parameters and handling shape constraints. The IT representation specifies a pattern of function forms: 

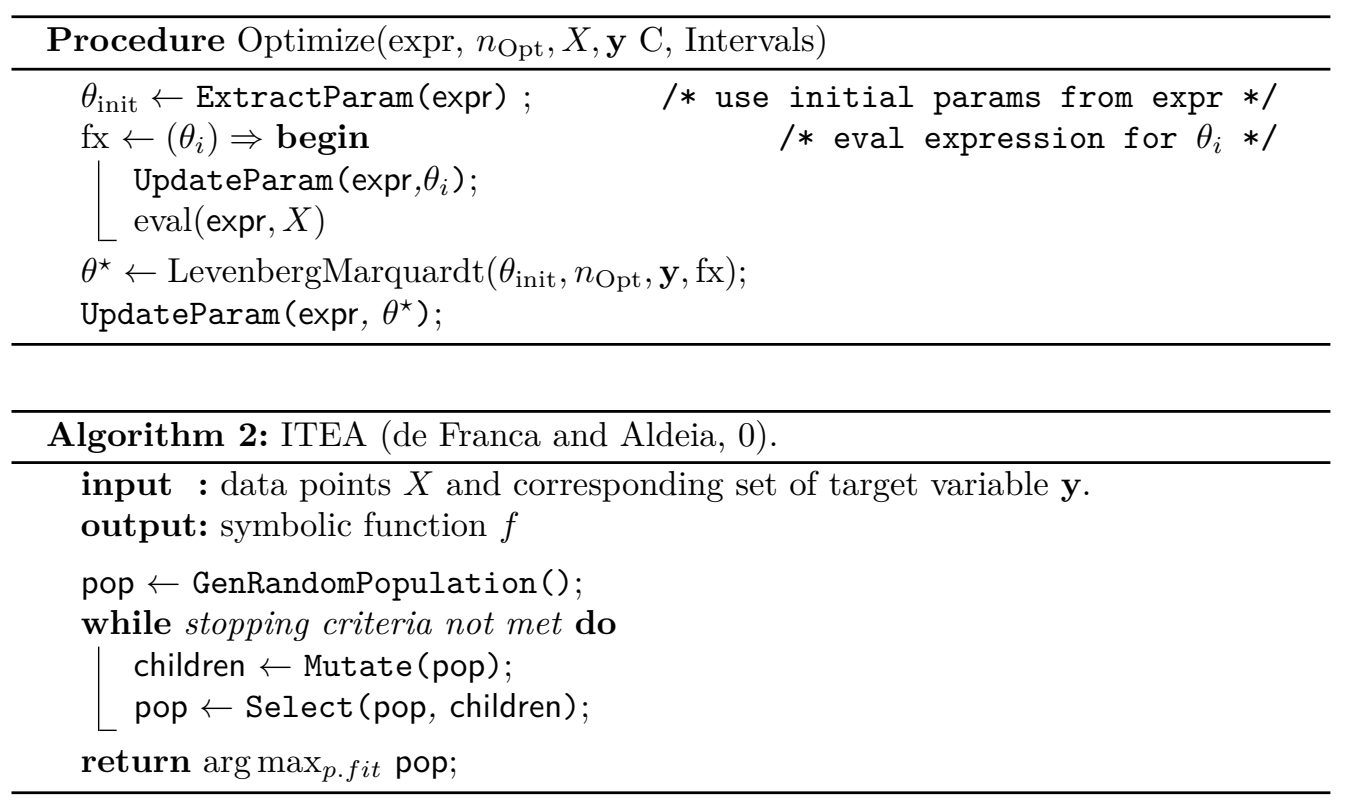

$$
\hat{f}(x)=\sum_{i} w_{i} \cdot t_{i}\left(\prod_{j=1}^{d} x_{j}^{k_{i j}}\right),
$$

where $w_{i}$ is the weight of the $i$-th term of the linear combination, $t_{i}$ is called a transformation function and can be any univariate function that is reasonable for the studied system and $k_{i j}$ is the strength of interaction for the $j$-th variable on the $i$-th term.

The algorithm is a simple mutation-based evolutionary algorithm depicted in Algorithm 2. It starts with a random population of expressions and repeats mutation and selection until convergence.

The mutation procedure chooses one of the following actions at random:

- Remove one random term of the expression.

- Add a new random term to the expression.

- Replace the interaction strengths of a random term of the expression.

- Replace a random term of the expression with the positive interaction of another random term.

- Replace a random term of the expression with the negative interaction of another random term.

The positive and negative interactions of terms is the sum or subtraction of the strengths of the variables. For example, if we have two terms $x_{1}^{2} x_{2}^{3}$ and $x_{1}^{-1} x_{2}^{1}$, and we want to apply the positive interaction to them, we would perform the operation $x_{1}^{2} \cdot x_{1}^{-1} \cdot x_{2}^{3} \cdot x_{2}^{1}$ that results in $x_{1} x_{2}^{4}$, similarly with the negative interactions we divide the first polynomial by the other, so performing the operation $x_{1}^{2} \cdot x_{1}^{1} \cdot x_{2}^{3} \cdot x_{2}^{-1}$, resulting in $x_{1}^{3} x_{2}^{2}$. 


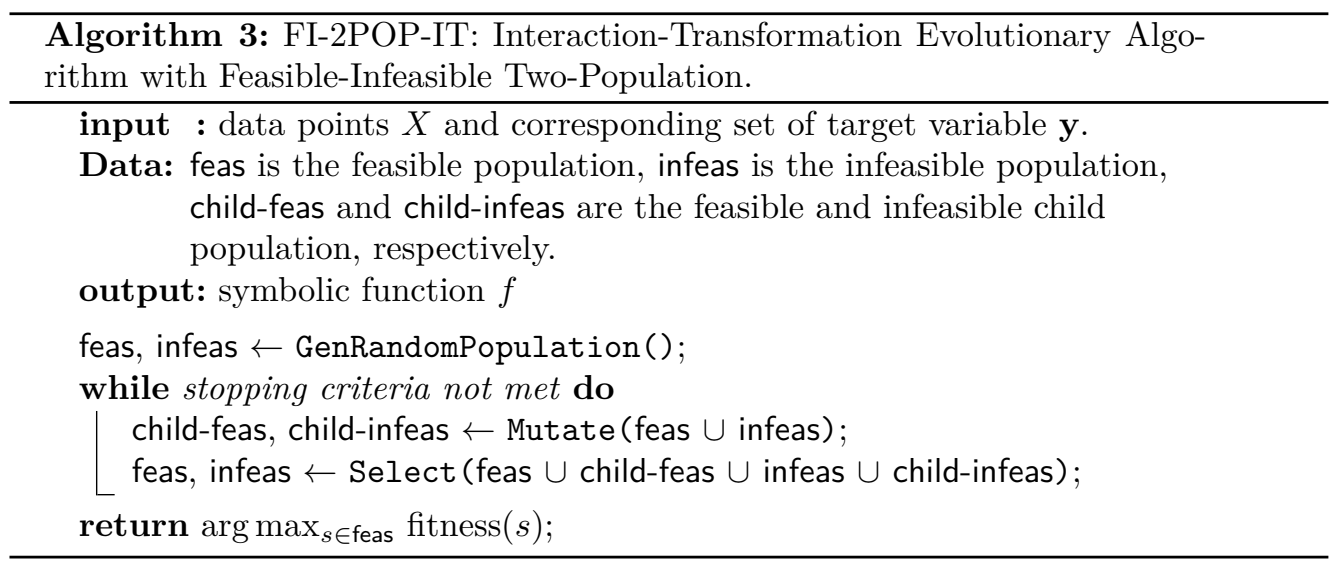

Weights within IT expressions can be efficiently optimized using ordinary least squares (OLS) after the mutation step.

This algorithm was reported to surpass different variants of GP and stay on par with nonlinear regression algorithms (de Franca and Aldeia, 0 ).

\subsection{Feasible-Infeasible Two-population with Interaction-Transformation}

In order introduce the shape-constraints into ITEA, we will use the approach called Feasible-Infeasible Two-population (FI-2POP) (Kimbrough et al., 2008) that works with two populations: one for feasible models and another for infeasible models. Despite its simplicity, it has been reported to work well on different problems (Liapis et al., 2015. Scirea et al., 2016, Covões and Hruschka, 2018). We have chosen this approach to deal with constraints for the ITEA algorithm since it does not demand fine tuning of a penalty function and a penalty coefficient, does not require any change to the original algorithm (as the repairing and constraint-aware operators), and does not introduce a high computational cost (i.e., multi-objective optimization).

Specifically for ITEA, the adaptation (named FI-2POP-IT) is depicted in Algorithm 3 The main difference from Algorithm 2 is that in FI-2POP-IT every function now produces two distinct populations, one for feasible solutions and another for the infeasible solutions. For example, the mutation operator when applied to an individual can produce either a feasible solution or an infeasible one, this new individual will be assigned to its corresponding population.

The fitness evaluation differs for each population, the feasible population minimizes the error function while the infeasible population minimizes the constraint violations.

\subsubsection{Defining Constraints with Interval Arithmetic}

The IT representation allows for a simple algorithm to calculate the $n$-th order partial derivatives of the expression and to perform IA. Additionally, only linear real-valued parameters are allowed for IT expressions. Therefore parameters can be optimized efficiently using ordinary least squares (OLS) and all shape constraints can be transformed to functions which are linear in the parameters.

Given an IT expression and the set of domains for each of the input variables, it is possible to calculate the image of the expression as the sum of the images of each term multiplied by their corresponding weight. The image of an interaction term $\operatorname{Im}($. is the product of the exponential of each variable interval $D($.$) with the corresponding$ 
strength:

$$
\operatorname{Im}(p(x))=\prod_{i=1}^{d} D\left(x_{i}\right)^{k_{i}},
$$

Arithmetic operators are well defined in interval arithmetic and the images of univariate non-linear functions can be easily determined.

The derivative of an IT expression can be calculated by the chain rule as follows $\left(g_{i}=t_{i} \circ p_{i}\right)$ :

$$
\begin{aligned}
\frac{\partial I T(x)}{\partial x_{j}} & =w_{1} \cdot g_{1}^{\prime}(x)+\ldots+w_{n} \cdot g_{n}^{\prime}(x) \\
\frac{\partial g_{i}(x)}{\partial x_{j}} & =t_{i}^{\prime}\left(p_{i}(x)\right) \cdot k_{j} \frac{p_{i}(x)}{x_{j}}
\end{aligned}
$$

All of the aforementioned transformation functions have derivatives representable by the provided functions. Following the same rationale the second order derivatives can be calculated as well.

\section{Experimental Setup}

For testing the effectiveness of the proposed approach we have created some artificial data sets generated from fluid dynamics engineering (FDE) taken from (Chen et al. 2018) and some selected physics models from the Feynman Symbolic Regression Databas $\oint^{2}$ (Udrescu and Tegmark, 2020) (FEY). Additionally, we have used data sets built upon real-world measurements from physical systems and engineering (RW). We have chosen these data sets because they are prototypical examples of non-trivial non-linear models which are relevant in practical applications.

For each of the problem instances we have defined a set of shape constraints that must be fulfilled by the SR solutions. For the FDE and FEY data sets, we have used constraints that can be derived from the known expressions over the given input space. For the physical systems we have defined the shape constraints based on expert knowledge. The input space and monotonicity constraints for all problem instances are given in the supplementary material.

In the following subsections we give more details on the problem instances including the constraint definitions and describe the methods to test the modified algorithms.

\subsection{Problem Instances}

All the data sets are described by the input variables names, the target variable, the true expression if it is known, domains of the variables for the training and test data, expected image of the function and monotonic constraints. All data set specifications are provided as a supplementary material.

Details for Aircraft Lift, Flow Psi, Fuel Flow models can be found in (Anderson. 2010, 1982). Additionally, we have selected a subset of the FEY problem instances (Udrescu and Tegmark 2020). The selection criteria was those problem instances which have been reported as unsolved without dimensional analysis or for which a required runtime of more than five minutes was reported by Udrescu and Tegmark (2020). We have not included the three benchmark problems from the closely related work of Bladek

\footnotetext{
${ }^{2}$ https://space.mit.edu/home/tegmark/aifeynman.html
} 
and Krawiec (2019), because we could easily solve them in preliminary experiments and additionally, the results would not be directly comparable because we do not support symmetry constraints.

All constraints for FDE and FYE have been determined by analysing the known expression and its partial derivatives. The formulas for the FDE and FYE problem instances are shown in Table 1 For all these problem instances we have generated data sets from the known formula by randomly sampling the input space using 100 data points for training and 100 data points for testing. For each of the data sets we generated two versions: one without noise and one where we have added normally distributed noise to the target variable $y^{\prime}=y+N\left(0,0.05 \sigma_{y}\right)$. Accordingly, the optimally achievable NMSE for the noisy problem instances is $0.25 \%$.

We have included three real-world data sets (RW). The dataset for Friction $\mu_{\text {stat }}$ and Friction $\mu_{\mathrm{dyn}}$ has been collected in a standardized testing procedure for friction plates and has two target variables that we model independently. The Flow Stress data set stems from hot compression tests of aluminium cylinders (Kabliman et al., 2019). The constraints for these data sets where set by specialists in the field.

The Cars data set has values for displacement, horsepower, weight, acceleration time and fuel efficiency of almost 400 different car models. This set was first used by Mammen et al. (2001) and then evaluated by Shah et al. (2016) to assess the performance of Support Vector Regression with soft constraints.

\subsection{Testing Methodology}

Each one of the following experiments has been repeated 30 times and, for every repetition, we have measured the NMSE in percent for both training and test data. The algorithms receive only the training set as the input and only the best found model is applied to the test set. Runs with and without shape constraints have been executed on the same hardware.

The first experiment tests the performance of the unmodified algorithms, i.e., without shape constraints. This test verifies whether unmodified SR algorithms identify conforming models solely from the observed data. If this result turns out negative, it means that constraint handling mechanisms are required for finding conforming models.

In the second experiment, we test the performance of the two modified algorithms. This will verify whether the algorithms are able to identify models conforming to prior knowledge encoded as shape constraints.

This testing approach allows us to compare the prediction errors (NMSE) of models produced with and without shape constraints to assess whether the prediction error improves or deteriorates. We perform the same analysis for the two groups of instances with and without noise separately.

\subsection{Algorithms Configurations}

In the following, the abbreviation GP refers to tree-based GP without local optimization and GPC refers to tree-based GP with local optimization. Both algorithms can be used with and without shape constraints. Table 2 shows the parameter values that have been used for the experiments with GP and GPC. ITEA refers to the InteractionTransformation Evolutionary Algorithm and FI-2POP-IT (short form: FIIT) refers to the two-population version of ITEA which supports shape constraints. Table 3 shows the parameters values for ITEA and FI-2POP-IT.

For comparison we have used auto-sklearn (Feurer et al. 2015) (AML) and an implementation of shape-constrained polynomial regression (SCPR) (Curmei and Hall 


\begin{tabular}{|c|c|}
\hline Name & Formula \\
\hline Aircraft lift & $C_{L}=C_{L \alpha}\left(\alpha+\alpha_{0}\right)+C_{L \delta_{e}} \delta_{e} \frac{S_{\mathrm{HT}}}{S_{\mathrm{ref}}}$ \\
\hline Flow psi & $\Psi=V_{\infty} r \sin \left(\frac{\theta}{2 \pi}\right)\left(1-\left(\frac{R}{r}\right)^{2}\right)+\frac{\Gamma}{2 \pi} \log \frac{r}{R}$ \\
\hline Fuel flow & $\dot{m}=\frac{p_{0} A \star}{\sqrt{T_{0}}} \sqrt{\frac{\gamma}{R}\left(\frac{2}{1+\gamma}\right)^{(\gamma+1) /(\gamma-1)}}$ \\
\hline Jackson 2.11 & $\frac{q}{4 \pi \epsilon y^{2}}\left(4 \pi \epsilon \operatorname{Volt} d-\frac{q d y^{3}}{\left(y^{2}-d^{2}\right)^{2}}\right)$ \\
\hline Wave power & $\frac{-32}{5} \frac{G^{4}}{c^{5}} \frac{(m 1 m 2)^{2}(m 1+m 2)}{r^{5}}$ \\
\hline I.6.20 & $\exp \left(\frac{-\left(\frac{\theta}{\sigma}\right)^{2}}{2}\right) \frac{1}{\sqrt{2 \pi} \sigma}$ \\
\hline I.9.18 & $\frac{G m 1 m 2}{(x 2-x 1)^{2}+(y 2-y 1)^{2}+(z 2-z 1)^{2}}$ \\
\hline I.15.3x & \\
\hline I.15.3t & $\left(t-\frac{u x}{c^{2}}\right) \frac{c^{2}}{\sqrt{1-\frac{u^{2}}{c^{2}}}}$ \\
\hline I.30.5 & $\operatorname{asin}\left(\frac{\operatorname{lambd}}{n d}\right)^{c^{2}}$ \\
\hline I.32.17 & $\frac{1}{2} \epsilon c E f^{2} \frac{8 \pi r^{2}}{3} \frac{\omega^{4}}{\left(\omega^{2}-\omega_{0}^{2}\right)^{2}}$ \\
\hline I. 41.16 & $\frac{h \omega^{3}}{\pi^{2} c^{2}\left(\exp \left(\frac{h \omega}{k b T}\right)-1\right)}$ \\
\hline I. 48.20 & $\frac{m c^{2}}{\sqrt{1-\frac{v^{2}}{c^{2}}}}$ \\
\hline II.6.15a & $\frac{\frac{p_{d}}{4 \pi \epsilon} 3 z}{r^{5}} \sqrt{x^{2}+y^{2}}$ \\
\hline II.11.27 & $\frac{n \alpha}{1-\frac{n \alpha}{3}} \epsilon E f$ \\
\hline II.11.28 & $1+\frac{n \alpha}{1-\frac{n \alpha}{3}}$ \\
\hline II.35.21 & $n_{r h o} m o m \tanh \left(\frac{m o m B}{k b T}\right)$ \\
\hline III.9.52 & $\frac{p_{d} E f t}{h} \sin \left(\frac{\left(\omega-\omega_{0}\right) t}{2}\right)^{2} \frac{1}{\left(\frac{\left(\omega-\omega_{0}\right) t}{2}\right)^{2}}$ \\
\hline III.10.19 & $m o m \sqrt{B x^{2}+B y^{2}+B z^{2}}$ \\
\hline
\end{tabular}

Table 1: Synthetic problem instances used for testing. The first three functions are from the FDE data sets, the rest from the FEY data sets.

$20203^{3}$ Auto-sklearn does not allow to set monotonicity constraints. Therefore, we can only use it as a comparison for symbolic regression models without constraints. We execute 30 independent repetitions for each problem with each run limited to one hour.

The degree for SCPR has been determined for each problem instance using 10fold cross-validation and using the best CV-RMSE as the selection criteria. We tested homogeneous polynomials up to degree eight (except for I.9.18 where we used a maximum degree of five because of runtime limits). The runtime for the grid search was limited to one hour for each problem instance. The final model is then trained with the selected degree on the full training set. SCPR is a deterministic algorithm therefore only a single run was required for each problem instance.

\footnotetext{
${ }^{3}$ We have initially also used XGboost, a popular implementation of gradient boosted trees, because it has previously been found that it compares well with symbolic regression methods (Orzechowski et al. 2018 and it supports monotonicity constraints. However, the results were much worse compared to the symbolic regression results which indicates that the method is not particularly well-suited for the problem instances in the benchmark set which are smooth and of low dimensionality.
} 


\begin{tabular}{ll} 
Parameter & Value \\
\hline Population size & 1000 \\
Generations & 200 \\
& 20 (for GPC with memetic optimization) \\
Initialization & PTC2 \\
$L_{\text {max }}, D_{\text {max }}$ & 50,20 \\
Fitness evaluation & NMSE with linear scaling \\
Selection & Tournament (group size $=5)$ \\
Crossover & Subtree crossover \\
Mutation (one of) & Replace subtree with random branch \\
& Add $x \sim N(0,1)$ to all numeric parameters \\
& Add $x \sim N(0,1)$ to a single numeric parameter \\
Crossover rate & Change a single function symbol \\
Mutation rate & $100 \%$ \\
Replacement & Generational with a single elite \\
Terminal set & real-valued parameters and input variables \\
Function set &,$+ * \%$, log, exp, sin, cos, tanh, $x^{2}, \sqrt{x}$ \\
GPC & max. 10 iterations of Levenberg-Marquardt $(\mathrm{LM})$ \\
\hline
\end{tabular}

Table 2: GP parameter configuration used for the experiments (\% refers to protected division). For the GPC runs we need fewer generations because of the faster convergence with local optimization.

\begin{tabular}{ll} 
Parameter & Value \\
\hline Population size & 200 \\
Number of Iterations & 500 \\
Function set & $\sin , \cos , \tanh , \sqrt{,} \log , \log 1 p$, exp \\
Fitness evaluation & RMSE \\
Maximum number of terms (init. pop.) & 4 \\
Range of strength values (init. pop.) & {$[-4,4]$} \\
Min. and max. term length & 2,6 \\
Regression model & OLS \\
\hline
\end{tabular}

Table 3: Parameter values for the ITEA algorithm that have been used for all experiments.

\section{Results}

In this section we report the obtained results in tabular and graphical forms focusing on the violations of the shape constraints, goodness of fit, computational overhead, and extrapolation capabilities with and without shape constraints.

\subsection{Constraint Violations}

The procedure for checking of constraint violations is as follows: for each problem instance we sample a million points uniformly from the full input space to produce a data set for checking constraint violations. The final SR solutions as well as their partial derivatives are then evaluated on this data set. If there is at least one point for which 


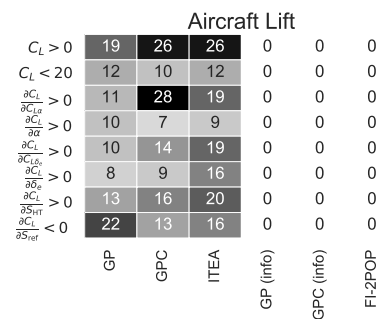

(a)

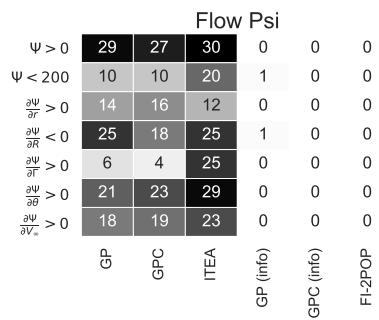

(b)

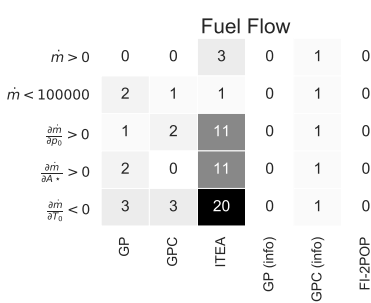

(c)

Figure 1: Constraint violation frequency for the solutions of every algorithm over the 30 executions for the FDE data sets. Only for the Fuel Flow problem a feasible solution can be identified even without shape constraints. FI-2POP always produces feasible solutions due to the nature of its constraint handling mechanism.

the shape constraints are violated then we count the SR solution as infeasible.

Figures 1a to 1c show exemplarily the number of infeasible SR models for each algorithm and constraint, over the 30 runs for the FDE data sets. The picture is similar for all problem instances. We observe that without shape constraints none of the algorithms produce feasible solutions in every run.

Only for the easiest data sets (e.g. Fuel Flow) we re-discovered the data-generating functions - which necessarily conform to the constraints - in multiple runs. Over all data sets, ITEA has the highest probability of producing infeasible models. We observe that even a small amount of noise increases the likelihood to produce infeasible models. In short, we can see that we cannot hope to find feasible solutions using SR algorithms without shape constraints for noisy data sets. This is consistent with the observation in (Bladek and Krawiec, 2019).

The results for the extended algorithms show, that with few exceptions, all three SR algorithms are capable of finding feasible solutions most of the time.

\subsection{Interpolation and Extrapolation}

The difference between SR models and shape-constrained SR models becomes clearer when we visualize their outputs.

Figures 2a to 2f show the predictions of every SR model identified for the Friction $\mu_{\text {stat }}$ data set. The partial dependence plots show the predictions for $\mu_{\text {stat }}$ for all 30 models over the complete range of values allowed for the two variables $p$ and $T$. The plot of $\mu_{\text {stat }}$ over $p$ shows the predictions when $v$ and $T$ are fixed to their median values and the plot of $\mu_{\text {stat }}$ over $T$ shows the predictions when $p$ and $v$ are fixed. The dashed vertical lines mark the subspace from which we have sampled points for the training and test data sets.

The algorithms without shape constraints produce extreme predictions when extrapolating (Figures 2a, 2c, 2e). For instance many of the functions have poles at a temperature $T$ close to zero which are visible in the plots as vertical lines. GP and GPC without shape constraints produced a few solutions which are wildly fluctuating over $p$ even within the interpolation range. Within the interpolation range ITEA produced the best SR solutions for the Friction data sets (see Figure $2 \mathrm{e}$ as well as Table 4). However, the models do not conform to prior knowledge as we would expect that $\mu_{\text {stat }}$ decreases with increasing pressure and temperature and the models show a slight increase in $\mu_{\text {stat }}$ 
when increasing $p$.

The model predictions for shape-constrained SR are shown in Figures 2b, 2d, and $2 \mathrm{f}$ The visualization clearly shows that there is higher variance and that all algorithms produced a few bad or even constant solutions. This invalidates our hypothesis that shape-constrained SR leads to improved predictive accuracy and instead indicates that there are convergence issues with our approach of including shape constraints. We will discuss this in more detail in the following sections. The visualization also shows that the solutions with shape constraints have better extrapolation behaviour and conform to shape constraints.

\subsection{Goodness-of-fit}

Table 4 shows the median NMSE of best solutions over 30 runs obtained by each algorithm, with and without shape constraints, on the test sets. The training results can be found in the supplementary material.

The table has 6 quadrants: the left block shows the results without side information, the right block shows the results with shape constraints; the top panel shows results for RW instances, the middle panel the results for the FDE and FYE instances, and the bottom panel the results for the same instances including $0.25 \%$ noise.

Analysing the detailed results we observe that the best result of all models with information is better for 18 of 42 instances (RW: 2, no noise: 6, noisy: 10). While the best result of models without information is better for 14 of 42 instances (RW: 2, no noise: 3 , noisy: 9).

Within both groups there are algorithms with significantly different test errors (without info: p-value: 0.0103, with info: p-value: $6.467 \cdot 10^{-6}$, Friedman's rank sum test with Davenport correction). Pairwise comparison of results without info shows that GPC is better than GP (p-value: 0.011) and AML (p-value: 0.043) using Friedman's rank sum test with Bergman correction. For the problem instances with noise AML produced the best result for only 1 out of 19 instances. For the instances without noise the results of AML are similar to results of GP, GPC, and ITEA. Pairwise comparison of results with info shows that SCPR is better than the other algorithms and no statistically significant difference was found between GP, GPC and FIIT. The p-values for all pairwise tests are shown in Table 5

Comparing the results with and without constraints for each algorithm individually, we find that the results are in general worse when using constraints. GP is better than GP (info) for 27 instances. GPC is better than GPC (info) for 32 instances. ITEA is better than FIIT for 19 instances. For the RW data sets, ITEA managed to find the best models on two out of the four instances. For Flow Stress, ITEA returned a solution that produced numerical errors for the test set. This is not the case when we include the shape-constraints, as we can see on the top-right quadrant. In this situation, FI2POP-IT was capable of finding expressions that did not return invalid results for the test set.

\subsection{Computational Overhead}

Another important impact of introducing constraints that should be considered is the computational overhead introduced to each approach.

For ITEA the execution time is approximately doubled when using the constraint handling. The reason being that parameter optimization is much easier for the ITEA representation and the calculation of the partial derivatives is a simple mechanical process as shown in Section 3.5.1. For GP and GPC the execution time factor is approximately 5 


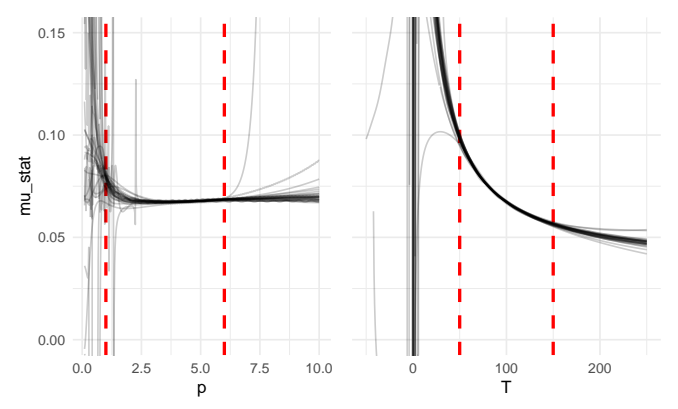

(a) GP

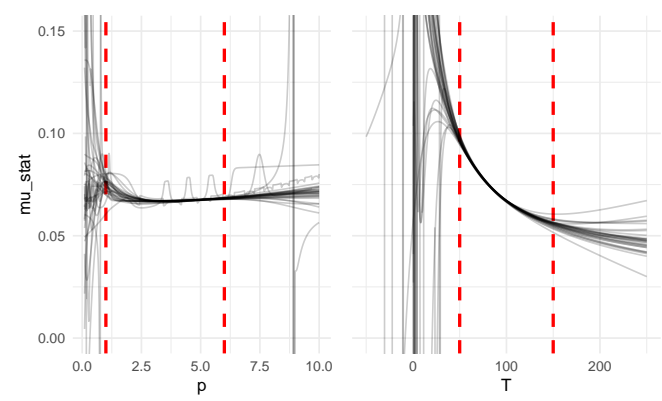

(c) GPC
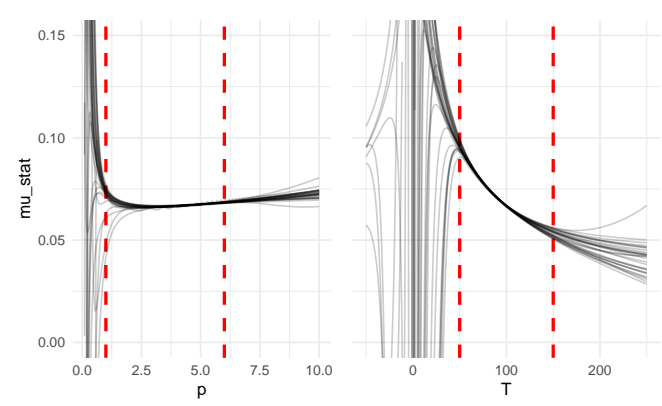

(e) ITEA

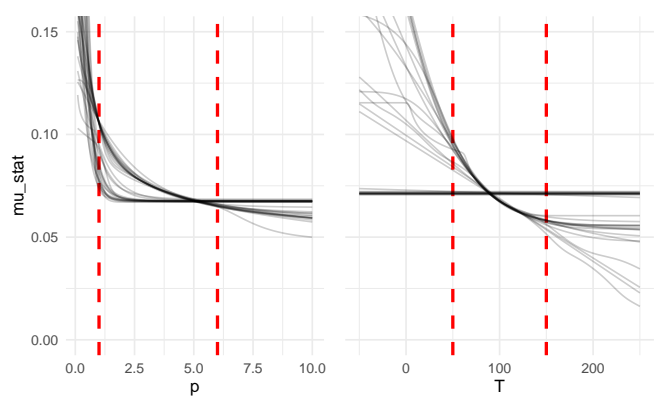

(b) GP (info)

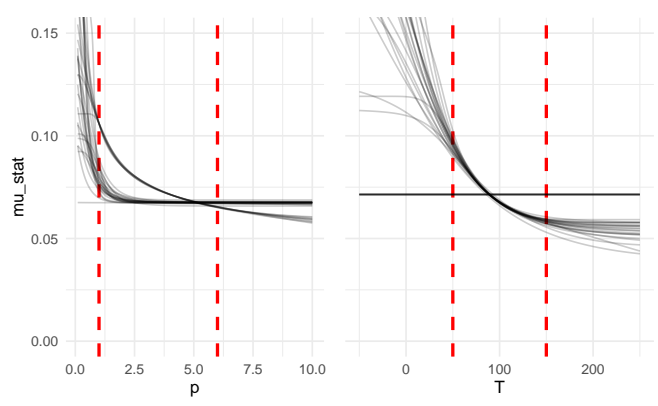

(d) GPC (info)

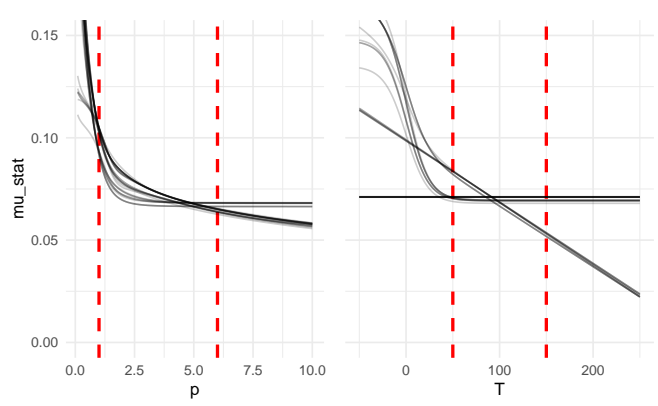

(f) FI-2POP-IT

Figure 2: Partial dependence plots for the Friction $\mu_{\text {stat }}$ models found by each algorithm over the 30 runs. Dashed lines mark the subspace from which training and test points were sampled. Algorithms with shape constraints (b, d, f) produce SR solutions which conform to prior knowledge and have better extrapolation behaviour but increased prediction error (cf. Table 4). 
Table 4: Median NMSE values for the test data. Values are multiplied by 100 (percentage) and truncated at the second decimal place.

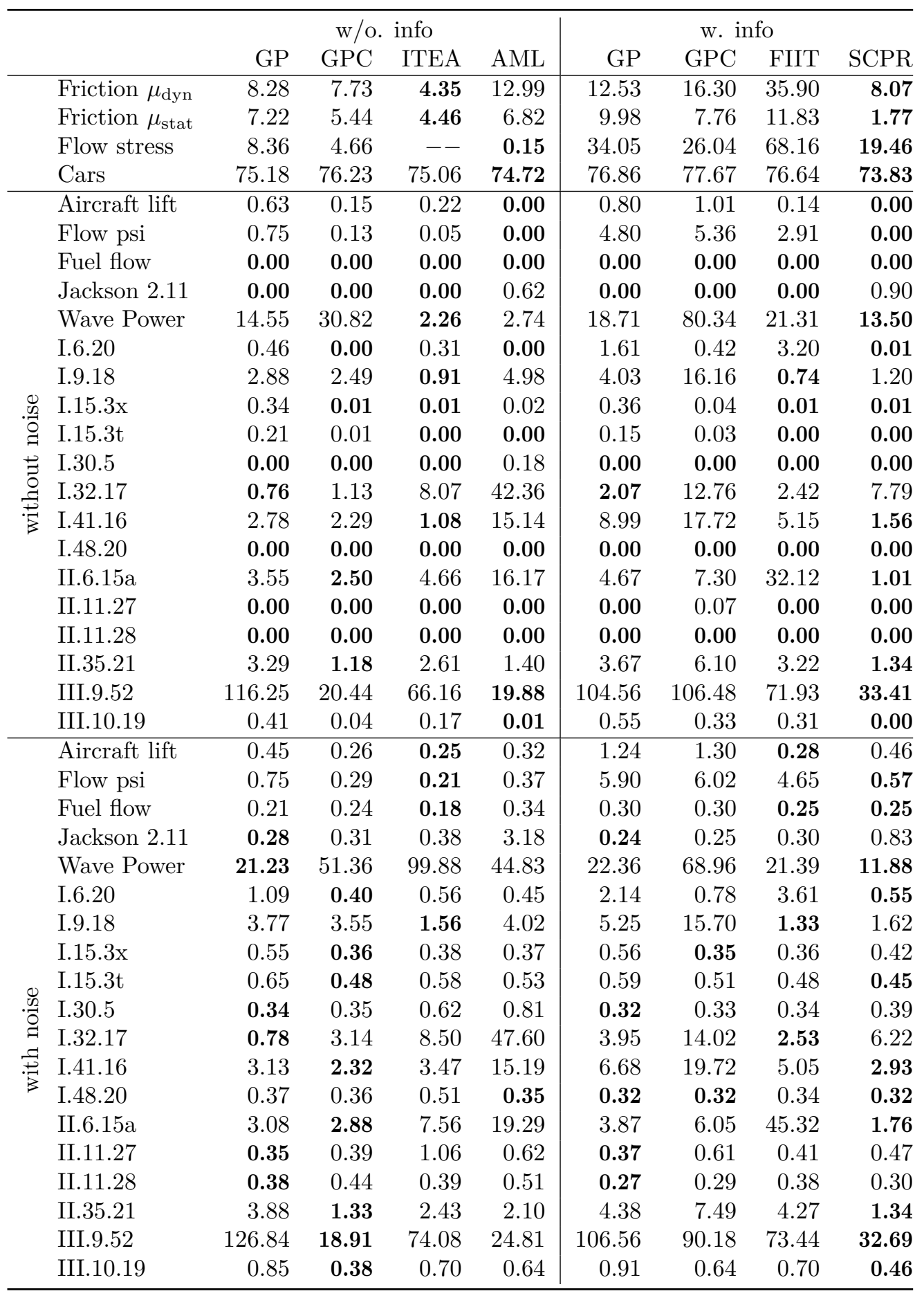




\begin{tabular}{lllllllll}
\multicolumn{4}{c}{ without info } & & \multicolumn{4}{c}{ with info } \\
GP & GPC & ITEA & AML & & GP & GPC & FIIT & SCPR \\
\hline n/a & $\mathbf{0 . 0 1 1}$ & 0.325 & 0.499 & & n/a & 0.151 & 0.353 & $\mathbf{0 . 0 0 2}$ \\
$\mathbf{0 . 0 1 1}$ & n/a & 0.325 & $\mathbf{0 . 0 4 3}$ & & 0.151 & n/a & 0.054 & $\mathbf{0 . 0 0 0}$ \\
0.325 & 0.325 & n $/ \mathrm{a}$ & 0.353 & & 0.353 & 0.054 & n/a & $\mathbf{0 . 0 2 8}$ \\
0.499 & $\mathbf{0 . 0 4 3}$ & 0.353 & n/a & & $\mathbf{0 . 0 0 2}$ & $\mathbf{0 . 0 0 0}$ & $\mathbf{0 . 0 2 8}$ & n/a
\end{tabular}

Table 5: p-values for pairwise comparison of algorithms in both groups (Friedman's rank sum test with Bergman correction)

when including shape constraints. The increased execution time for GP results from the additional effort for building the partial derivatives for each solution candidate and for the interval evaluation. We observed that the increase in execution time is less extreme for problem instances with a large number of rows where the relative effort for symbolic derivation of solution candidates becomes smaller.

\section{Discussion}

The results presented on the previous section largely corroborate our initial assumptions for shape-constrained symbolic regression. First of all, when we do not explicitly consider shape constraints within SR algorithms we are unlikely to find solutions which conform to expected behaviour. We showed that the results produced by the two newly-introduced algorithms in fact conform to shape constraints. Our assessment of the extrapolation and interpolation behaviour of SR models highlighted the bad extrapolation behaviour as well as occasional problems even for interpolation. The improved results when including shape constraints support the argument to include interval arithmetic to improve the robustness of SR solutions (Keijzer, 2003 Pennachin et al. 2010 ).

However, our results also show that including shape-constraints via interval arithmetic leads to SR solutions with higher prediction errors on training and test sets. While the increased error on the training set is expected, we hoped we would be able to improve prediction errors on the test set. Assuming that the constraints are correct, this should hypothetically be possible because the shape constraints provide additional information for improving the fit on noisy data sets. In fact, we observed that the results got worse when using information for tree-based GP with and without local optimization (GPC). There are several possible explanations such as slower convergence, more rapid loss of diversity, or rejection of feasible solutions because of the pessimistic bounds produced by interval arithmetic. Another hypothesis is that the positive effect of shape constraints becomes more relevant with higher noise levels. We are not able to give a conclusive answer for the main cause of the higher prediction errors with side information and leave this question open for future research.

Comparison with AutoML as implemented by auto-sklearn showed that GP with parameter optimization (GPC) produced better test results than AutoML (p-value: 0.011) over the benchmark set without shape-constraints. However, AutoML does not support monotonicity constraints and, because of that, we cannot use it to compare with the results using side information. Therefore, we compared the results of our proposed algorithms with shape-constrained polynomial regression (SCPR). The results show that SCPR performs better than the evolutionary algorithms for this benchmark set, which indicates that we can find a good approximation of many of our benchmark instances using polynomials. 
An advantage of SCPR is that it is formulated as a convex optimization problem that can be solved efficiently and deterministically by highly-tuned solvers. A drawback of SCPR is the potentially large model size. The number of terms of a homogeneous $n$ variate polynomial of degree $d$ is $\left(\begin{array}{c}n+d \\ n\end{array}\right)$. Our experiments found that polynomial degrees of up to eight were required to find a good fit. The studied problems had five variables on average, which led to more than a thousand terms in our polynomial models. The models produced by the evolutionary algorithms were, on average, much smaller as we used a limit of 50 nodes for GP expression trees and 6 terms for ITEA/FI-2POP-IT.

\section{Conclusions}

In this paper we have introduced shape-constrained symbolic regression which allows to include prior knowledge into SR. Shape-constrained symbolic regression allows to enforce that the model output must be within given bounds, or that outputs must be monotonically increasing or decreasing over selected inputs. The structure and the parameters of the symbolic regression model are however still identified by the algorithm.

We have described two algorithms for shape-constrained symbolic regression which are extensions of tree-based genetic programming with optional local optimization and the Interaction-Transformation Evolutionary Algorithm that uses a more restrictive representation with the goal of returning simpler expressions.

The extensions add the ability to calculate partial derivatives for any expression generated by the algorithms and use interval arithmetic to determine bounds and determine if models conform to shape constrains. Two approaches for handling constraint violations have been tested. The first approach simply adjusts fitness for infeasible solutions while the second approach splits the population into feasible and infeasible solutions.

The results showed the importance of treating the shape constraints inside the algorithms. First of all, we have collected more evidence that without any feasibility control, is unlikely to find feasible solutions for most of the problems. Following, we verified the efficacy of our approach by measuring the frequency of infeasible solutions and reporting the median numerical error of our models. The modified algorithms were all capable of finding models conforming to the shape constraints. This shows that the introduction of shape constraints can help us finding more realistic models. However, we have also found that the extended algorithms with shape constraints produce worse solutions on the test set on average.

For the next steps we intend to analyse in detail the causes for the worse solutions with shape-constrained SR. The bounds determined via interval arithmetic are very wide and might lead to rejection of feasible solutions as well as premature convergence. This is an issue that could potentially be solved by using more elaborate bound estimation schemes such as affine arithmetic or recursive splitting. Other possibilities for the constraint-handling include multi-objective optimization and penalty functions. Alternatively, optimistic approaches (e.g. using sampling) or a hybridization of pessimistic and optimistic approaches for shape-constrained regression can be used to potentially improve the results. Additionally, it would be worthwhile to study the effects of constraint-handling mechanisms on population diversity in more detail.

\section{Acknowledgments}

This project is partially funded by Fundação de Amparo à Pesquisa do Estado de São Paulo (FAPESP), grant number 2018/14173-8. And some of the experiments (ITEA) made use of the Intel®AI DevCloud, which Intel®provided free access. 
The authors gratefully acknowledge support by the Christian Doppler Research Association and the Federal Ministry of Digital and Economic Affairs within the Josef Ressel Centre for Symbolic Regression.

\section{References}

Ahmadi, A. A. and Majumdar, A. (2019). DSOS and SDSOS optimization: More tractable alternatives to sum of squares and semidefinite optimization. SIAM Journal on Applied Algebra and Geometry, 3(2):193-230.

Anderson, J. D. (1982). Modern Compressible Flow: With Historical Perspective (McGraw-Hill series in mechanical engineering). McGraw-Hill New York.

Anderson, J. D. (2010). Fundamentals of Aerodynamics. McGraw-Hill Education, 5th edition.

Arnaldo, I., Krawiec, K., and O'Reilly, U.-M. (2014). Multiple regression genetic programming. In Proceedings of the 2014 Annual Conference on Genetic and Evolutionary Computation, GECCO '14, pages 879-886, New York, NY, USA. ACM.

Bladek, I. and Krawiec, K. (2019). Solving symbolic regression problems with formal constraints. In Proceedings of the Genetic and Evolutionary Computation Conference, pages $977-984$.

Castillo, F., Marshall, K., Green, J., and Kordon, A. (2003). A methodology for combining symbolic regression and design of experiments to improve empirical model building. In Genetic and Evolutionary Computation Conference, pages 1975-1985. Springer.

Castillo, F. A., Villa, C. M., and Kordon, A. K. (2013). Symbolic regression model comparison approach using transmitted variation. In Genetic Programming Theory and Practice X, pages 139-154. Springer.

Chen, C., Luo, C., and Jiang, Z. (2018). A multilevel block building algorithm for fast modeling generalized separable systems. Expert Systems with Applications, 109:25-34.

Covões, T. F. and Hruschka, E. R. (2018). Classification with multi-modal classes using evolutionary algorithms and constrained clustering. In 2018 IEEE Congress on Evolutionary Computation (CEC), pages 1-8. IEEE.

Curmei, M. and Hall, G. (2020). Shape-constrained regression using sum of squares polynomials.

de França, F. O. (2018). A greedy search tree heuristic for symbolic regression. Information Sciences, 442:18-32.

de Franca, F. O. and Aldeia, G. S. I. (0). Interaction-transformation evolutionary algorithm for symbolic regression. Evolutionary Computation, 0(ja):1-25. PMID: 33306435 .

Dick, G. (2017). Revisiting interval arithmetic for regression problems in genetic programming. In Proceedings of the Genetic and Evolutionary Computation Conference Companion, pages 129-130. ACM. 
Ferreira, C. (2001). Gene expression programming: A new adaptive algorithm for solving problems. Complex Systems, 13(2):87-129.

Feurer, M., Klein, A., Eggensperger, K., Springenberg, J., Blum, M., and Hutter, F. (2015). Efficient and robust automated machine learning. In Cortes, C., Lawrence, N. D., Lee, D. D., Sugiyama, M., and Garnett, R., editors, Advances in Neural Information Processing Systems 28, pages 2962-2970. Curran Associates, Inc.

Fortin, F.-A., Rainville, F.-M. D., Gardner, M.-A., Parizeau, M., and Gagné, C. (2012). Deap: Evolutionary algorithms made easy. Journal of Machine Learning Research, 13(Jul):2171-2175.

Guntuboyina, A., Sen, B., et al. (2018). Nonparametric shape-restricted regression. Statistical Science, 33(4):568-594.

Gupta, M., Cotter, A., Pfeifer, J., Voevodski, K., Canini, K., Mangylov, A., Moczydlowski, W., and van Esbroeck, A. (2016). Monotonic calibrated interpolated look-up tables. Journal of Machine Learning Research, 17(109):1-47.

Hall, G. (2018). Optimization over nonnegative and convex polynomials with and without semidefinite programming. PhD thesis, Princeton University.

Hickey, T., Ju, Q., and Van Emden, M. H. (2001). Interval arithmetic: From principles to implementation. Journal of the ACM (JACM), 48(5):1038-1068.

Houck, C. R., Joines, J. A., Kay, M. G., and Wilson, J. R. (1997). Empirical investigation of the benefits of partial lamarckianism. Evolutionary Computation, 5(1):31-60.

Kabliman, E., Kolody, A. H., Kommenda, M., and Kronberger, G. (2019). Prediction of stress-strain curves for aluminium alloys using symbolic regression. AIP Conference Proceedings, 2113(1):180009.

Keijzer, M. (2003). Improving symbolic regression with interval arithmetic and linear scaling. In European Conference on Genetic Programming, pages 70-82. Springer.

Kim, S., Lu, P. Y., Mukherjee, S., Gilbert, M., Jing, L., Ceperic, V., and Soljačić, M. (2019). Integration of neural network-based symbolic regression in deep learning for scientific discovery. ArXiv, abs/1912.04825.

Kimbrough, S. O., Koehler, G. J., Lu, M., and Wood, D. H. (2008). On a feasibleinfeasible two-population (fi-2pop) genetic algorithm for constrained optimization: Distance tracing and no free lunch. European Journal of Operational Research, 190(2):310-327.

Kommenda, M., Burlacu, B., Kronberger, G., and Affenzeller, M. (2020). Parameter identification for symbolic regression using nonlinear least squares. Genetic Programming and Evolvable Machines, Special Issue on Integrating Numerical Optimization Methods with Genetic Programming, pages 1-31.

Koza, J. R. (1992). Genetic programming: on the programming of computers by means of natural selection, volume 1 . MIT press.

Kurse, M. U., Lipson, H., and Valero-Cuevas, F. J. (2012). Extrapolatable analytical functions for tendon excursions and moment arms from sparse datasets. IEEE Transactions on Biomedical Engineering, 59(6):1572-1582. 
Li, L., Fan, M., Singh, R., and Riley, P. (2019). Neural-guided symbolic regression with semantic prior. arXiv preprint arXiv:1901.07714.

Liapis, A., Holmgård, C., Yannakakis, G. N., and Togelius, J. (2015). Procedural personas as critics for dungeon generation. In European Conference on the Applications of Evolutionary Computation, pages 331-343. Springer.

Ljung, L. (2010). Perspectives on system identification. Annual Reviews in Control, $34(1): 1-12$.

Luke, S. (2000). Two fast tree-creation algorithms for genetic programming. Evolutionary Computation, IEEE Transactions on, 4:274-283.

Mammen, E., Marron, J., Turlach, B., Wand, M., et al. (2001). A general projection framework for constrained smoothing. Statistical Science, 16(3):232-248.

McConaghy, T. (2011). FFX: Fast, scalable, deterministic symbolic regression technology. In Genetic Programming Theory and Practice IX, pages 235-260. Springer.

Miller, J. F. and Harding, S. L. (2008). Cartesian genetic programming. In Proceedings of the 10th annual conference companion on Genetic and evolutionary computation, pages 2701-2726. ACM.

Moraglio, A., Krawiec, K., and Johnson, C. G. (2012). Geometric semantic genetic programming. In International Conference on Parallel Problem Solving from Nature, pages 21-31. Springer.

Oltean, M. and Grosan, C. (2003). A comparison of several linear genetic programming techniques. Complex Systems, 14(4):285-314.

O'Neill, M. and Ryan, C. (2001). Grammatical evolution. IEEE Transactions on Evolutionary Computation, 5(4):349-358.

Orzechowski, P., La Cava, W., and Moore, J. H. (2018). Where are we now? a large benchmark study of recent symbolic regression methods. In Proceedings of the Genetic and Evolutionary Computation Conference, pages 1183-1190.

Papp, D. and Alizadeh, F. (2014). Shape-constrained estimation using nonnegative splines. Journal of Computational and graphical Statistics, 23(1):211-231.

Pawlak, T. P. and Krawiec, K. (2018). Competent geometric semantic genetic programming for symbolic regression and boolean function synthesis. Evolutionary Computation, 26(2):177-212. PMID: 28207295.

Pennachin, C. L., Looks, M., and de Vasconcelos, J. A. (2010). Robust symbolic regression with affine arithmetic. In Proceedings of the 12th Annual Conference on Genetic and Evolutionary Computation, pages 917-924. ACM.

Petersen, B. K., Larma, M. L., Mundhenk, T. N., Santiago, C. P., Kim, S. K., and Kim, J. T. (2019). Deep symbolic regression: Recovering mathematical expressions from data via risk-seeking policy gradients. arXiv preprint arXiv:1912.04871.

Ruberto, S., Terragni, V., and Moore, J. H. (2020). Sgp-dt: Semantic genetic programming based on dynamic targets. In Hu, T., Lourenço, N., Medvet, E., and Divina, F., editors, Genetic Programming, pages 167-183, Cham. Springer International Publishing. 
Rudin, C. (2019). Stop explaining black box machine learning models for high stakes decisions and use interpretable models instead. Nature Machine Intelligence, 1(5):206215.

Sahoo, S. S., Lampert, C. H., and Martius, G. (2018). Learning equations for extrapolation and control. arXiv preprint arXiv:1806.07259.

Schmidt, M. D. and Lipson, H. (2009). Incorporating expert knowledge in evolutionary search: a study of seeding methods. In Proceedings of the 11th Annual conference on Genetic and evolutionary computation, pages 1091-1098. ACM.

Scirea, M., Togelius, J., Eklund, P., and Risi, S. (2016). Metacompose: A compositional evolutionary music composer. In International Conference on Computational Intelligence in Music, Sound, Art and Design, pages 202-217. Springer.

Shah, S., Sardeshmukh, A., Ahmed, S., and Reddy, S. (2016). Soft monotonic constraint support vector regression. In COMAD, pages 64-73.

Smits, G. F. and Kotanchek, M. (2005). Pareto-front exploitation in symbolic regression. In Genetic programming theory and practice II, pages 283-299. Springer.

Stewart, R. and Ermon, S. (2017). Label-free supervision of neural networks with physics and domain knowledge. In Thirty-First AAAI Conference on Artificial Intelligence, AAAI'17, pages 2576-2582. AAAI Press.

Sun, H. and Moscato, P. (2019). A memetic algorithm for symbolic regression. In 2019 IEEE Congress on Evolutionary Computation (CEC), pages 2167-2174.

Tibshirani, R. J., Hoefling, H., and Tibshirani, R. (2011). Nearly-isotonic regression. Technometrics, 53(1):54-61.

Topchy, A. and Punch, W. F. (2001). Faster genetic programming based on local gradient search of numeric leaf values. In Spector, L., Goodman, E. D., Wu, A., Langdon, W. B., Voigt, H.-M., Gen, M., Sen, S., Dorigo, M., Pezeshk, S., Garzon, M. H., and Burke, E., editors, Proceedings of the Genetic and Evolutionary Computation Conference (GECCO-2001), pages 155-162, San Francisco, California, USA. Morgan Kaufmann.

Udrescu, S.-M. and Tegmark, M. (2020). AI Feynman: A physics-inspired method for symbolic regression. Science Advances, 6(16):eaay2631.

Versino, D., Tonda, A., and Bronkhorst, C. A. (2017). Data driven modeling of plastic deformation. Computer Methods in Applied Mechanics and Engineering, 318:9811004 .

Worm, T. and Chiu, K. (2013). Prioritized grammar enumeration: symbolic regression by dynamic programming. In Proceedings of the 15th annual conference on Genetic and evolutionary computation, pages 1021-1028. ACM.

Wright, I. W., Wegman, E. J., et al. (1980). Isotonic, convex and related splines. The Annals of Statistics, 8(5):1023-1035.

Zhu, Y., Zabaras, N., Koutsourelakis, P.-S., and Perdikaris, P. (2019). Physicsconstrained deep learning for high-dimensional surrogate modeling and uncertainty quantification without labeled data. Journal of Computational Physics, 394:56-81. 


\begin{tabular}{|c|c|c|}
\hline Name & Input space & Constraints \\
\hline Aircraft lift & $\begin{array}{c}\left(C_{L \alpha}, \alpha, C_{L \delta_{e}}, \delta_{e}, S_{\mathrm{HT}}, S_{\mathrm{ref}}\right) \\
\in[0.3 . .0 .9] \times[2 . .12] \times[0.3 . .0 .9] \times[0 . .12] \times[0.5 . .2] \times[3 . .10]\end{array}$ & $(1,1,1,1,1,-1)$ \\
\hline Flow psi & $\begin{aligned} &\left(V_{\infty}, R, \Gamma, r, \theta\right) \\
& \epsilon[30 . .100] \times[0.1 . .0 .5] \times[2 . .15] \times[0.5 . .1 .5] \times[10 . .90]\end{aligned}$ & $(1,1,1,-1,1)$ \\
\hline Fuel flow & $\begin{array}{c}\left(A \star, p_{0}, T_{0}\right) \\
\in[0.2 . .2] \times\left[3 \cdot 10^{5} . .7 \cdot 10^{5}\right] \times[200 . .400]\end{array}$ & $(1,1,-1)$ \\
\hline Jackson 2.11 & $\begin{array}{c}(q, y, \text { Volt }, d, \epsilon) \\
\in[1 . .5] \times[1 . .3] \times[1 . .5] \times[4 . .6] \times[1 . .5]\end{array}$ & $(1,-1,1,1,1)$ \\
\hline Wave power & $\begin{aligned} &(G, c, m 1, m 2, r) \\
& \in[1 . .2] \times[1 . .2] \times[1 . .5] \times[1 . .5] \times[1 . .2]\end{aligned}$ & $(-1,1,-1,-1,1)$ \\
\hline I. 6.20 & $(\sigma, \theta) \in[1 . .3]^{2}$ & $(0,-1)$ \\
\hline I.9.18 & $\begin{array}{c}(m 1, m 2, G, x 1, x 2, y 1, y 2, z 1, z 2) \\
\in[1 . .2] \times[1 . .2] \times[1 . .2] \times[3 . .4] \\
\times[1 . .2] \times[3 . .4] \times[1 . .2] \times[3 . .4] \times[1 . .2]\end{array}$ & $\begin{array}{l}(1,1,1,-1,1 \\
-1,1,-1,1)\end{array}$ \\
\hline I.15.3x & $(x, u, c, t) \in[5 . .10] \times[1 . .2] \times[3 . .20] \times[1 . .2]$ & $(1,0,-1,-1)$ \\
\hline I.15.3t & $(x, c, u, t) \in[1 . .5] \times[3 . .10] \times[1 . .2] \times[1 . .5]$ & $(0,0,0,1)$ \\
\hline I.30.5 & $(l a m b d, d, n) \in[1 . .5] \times[2 . .5] \times[1 . .5]$ & $(1,-1,-1)$ \\
\hline I. 32.17 & $\begin{array}{c}\left(\epsilon, c, E f, r, \omega, \omega_{0}\right) \\
\in[1 . .2] \times[1 . .2] \times[1 . .2] \times[1 . .2] \times[1 . .2] \times[3 . .5]\end{array}$ & $(1,1,1,1,1,-1)$ \\
\hline I.41.16 & $(\omega, T, h, k b, c) \in[1 . .5]^{5}$ & $(0,1,-1,1,-1)$ \\
\hline I. 48.20 & $(m, v, c) \in[1 . .5] \times[1 . .2] \times[3 . .20]$ & $(1,1,1)$ \\
\hline II. $6.15 \mathrm{a}$ & $\left(\epsilon, p_{d}, r, x, y, z\right) \in[1 . .3]^{6}$ & $(-1,1,-1,1,1,1)$ \\
\hline II.11.27 & $(n, \alpha, \epsilon, E f) \in[0 . .1] \times[0 . .1] \times[1 . .2] \times[1 . .2]$ & $(1,1,1,1)$ \\
\hline II.11.28 & $(n$, alpha $) \in[0 . .1]^{2}$ & $(1,1)$ \\
\hline II. 35.21 & $\left(n_{r h o}, \operatorname{mom}, B, k b, T\right) \in[1 . .5]^{5}$ & $(1,1,1,-1,-1)$ \\
\hline III.9.52 & $\begin{array}{l}\left(p_{d}, E f, t, h, \omega, \omega_{0}\right) \\
\in[1-3] \times[1 \quad 3] \times[1-3] \times[1,3] \times[1,5] \times[1,5]\end{array}$ & $(1,1,0,-1,0,0)$ \\
\hline III.10.19 & $($ mom, Bx $, B y, B z) \in[1 . .5]^{4}$ & $(1,1,1,1)$ \\
\hline Friction $\mu_{\text {dyn }}$ & $(p, v, T) \in[0.1 . .15] \times[0.01 . .3] \times[-50 . .250]$ & $(-1,-1,-1)$ \\
\hline Friction $\mu_{\text {stat }}$ & $(p, v, T) \in[0.1 . .15] \times[0.01 . .3] \times[-50 . .250]$ & $(-1,0,-1)$ \\
\hline Flow stress & $(\phi, \dot{\phi}, T) \in[0 . .1] \times[0.001 . .10] \times[250 . .600]$ & $(0,1,-1)$ \\
\hline Cars & $\begin{array}{c}(c y l, d i s, h p, w, a c c) \\
\in[3 . .8] \times[68 . .455] \times[46 . .230] \times[8 . .24 .8]\end{array}$ & $(0,-1,-1,-1,0)$ \\
\hline
\end{tabular}

Table 6: Input space and the monotonicity constraints we used for all benchmark instances. In the constraints tuple a negative value for $c_{i}$ means that the model is nonincreasing over the $i$-th variable, a positive value means that the model is non-decreasing and a zero means that there is no monotonicity constraint.

\section{Supplementary}

\subsection{Problem definition details}

Table 6 shows the input space and the monotonicity constraints that we have used for the problem instances.

\subsection{Training results}

Table 7 shows the median NMSE values (in percent) on the training set for the best solutions over 30 runs of each algorithm. The test results are given in the paper.

\subsection{Sensitivity to population size}

For the experiments in the paper we have used parameter settings based on prior knowledge about settings which tend to produce good results on a wide range of problems. For instance we have set the population size to 1000 individuals for GP and 200 for ITEA and FIIT. Similarly, we have chosen other parameter values. It should be noted that we have not tried to tune the hyper-parameters (globally or for individual instances). Since 
Table 7: Median of the NMSE for the training data without and with shape constraints.

Values are multiplied by 100 (percentage) and truncated at the second decimal place.

\begin{tabular}{|c|c|c|c|c|c|c|c|c|c|}
\hline & \multicolumn{4}{|c|}{ w/o. info } & \multicolumn{4}{|c|}{ w. info } \\
\hline & & GP & GPC & ITEA & AML & GP & GPC & FIIT & SCPR \\
\hline \multirow{23}{*}{ 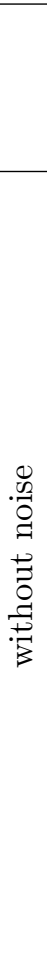 } & Friction $\mu_{\text {dyn }}$ & 0.80 & 0.48 & 0.24 & 0.07 & 2.02 & 1.51 & 1.98 & 3.05 \\
\hline & Friction $\mu_{\text {stat }}$ & 0.41 & 0.30 & 0.25 & 0.11 & 1.84 & 0.95 & 5.81 & 0.61 \\
\hline & Flow stress & 4.73 & 4.85 & 4.70 & 0.05 & 23.57 & 22.76 & 42.55 & 14.27 \\
\hline & Cars & 9.34 & 10.78 & 7.48 & 6.42 & 10.79 & 11.26 & 11.56 & 9.78 \\
\hline & Aircraft lift & 0.33 & 0.08 & 0.08 & 0.00 & 0.49 & 0.68 & 0.13 & 0.00 \\
\hline & Flow psi & 0.54 & 0.14 & 0.02 & 0.00 & 4.54 & 4.59 & 3.63 & 0.00 \\
\hline & Fuel flow & 0.00 & 0.00 & 0.00 & 0.00 & 0.00 & 0.00 & 0.00 & 0.00 \\
\hline & Jackson 2.11 & 0.00 & 0.00 & 0.00 & 0.10 & 0.00 & 0.00 & 0.00 & 0.00 \\
\hline & Wave Power & 0.34 & 0.69 & 0.01 & 0.41 & 1.07 & 6.88 & 0.93 & 0.00 \\
\hline & I. 6.20 & 0.18 & 0.00 & 0.01 & 0.00 & 1.47 & 0.34 & 3.61 & 0.00 \\
\hline & I.9.18 & 0.94 & 0.63 & 0.17 & 0.47 & 1.15 & 4.48 & 0.18 & 0.00 \\
\hline & I.15.3x & 0.18 & 0.00 & 0.00 & 0.00 & 0.19 & 0.01 & 0.00 & 0.00 \\
\hline & I.15.3t & 0.11 & 0.00 & 0.00 & 0.00 & 0.10 & 0.01 & 0.00 & 0.00 \\
\hline & I.30.5 & 0.00 & 0.00 & 0.00 & 0.22 & 0.00 & 0.00 & 0.00 & 0.00 \\
\hline & I.32.17 & 0.18 & 0.13 & 0.05 & 20.19 & 0.43 & 4.89 & 0.06 & 0.11 \\
\hline & I.41.16 & 0.19 & 0.18 & 0.03 & 12.11 & 1.13 & 1.52 & 0.24 & 0.00 \\
\hline & I. 48.20 & 0.00 & 0.00 & 0.00 & 0.00 & 0.00 & 0.00 & 0.00 & 0.00 \\
\hline & II.6.15a & 0.64 & 0.15 & 0.18 & 19.98 & 1.16 & 1.24 & 0.61 & 0.00 \\
\hline & II.11.27 & 0.00 & 0.00 & 0.00 & 0.00 & 0.00 & 0.04 & 0.00 & 0.00 \\
\hline & II.11.28 & 0.00 & 0.00 & 0.00 & 0.00 & 0.00 & 0.00 & 0.00 & 0.00 \\
\hline & II.35.21 & 1.75 & 0.49 & 0.44 & 0.17 & 2.43 & 3.95 & 2.08 & 0.00 \\
\hline & III.9.52 & 23.69 & 7.14 & 8.13 & 5.44 & 32.27 & 32.56 & 21.45 & 0.20 \\
\hline & III.10.19 & 0.31 & 0.02 & 0.08 & 0.00 & 0.37 & 0.23 & 0.21 & 0.00 \\
\hline \multirow{19}{*}{ 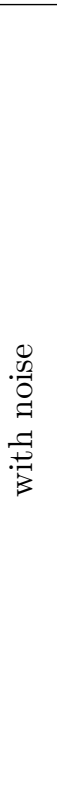 } & Aircraft lift & 0.45 & 0.26 & 0.25 & 0.22 & 0.76 & 0.87 & 0.28 & 3.94 \\
\hline & Flow psi & 0.75 & 0.29 & 0.21 & 0.21 & 5.69 & 5.22 & 4.65 & 4.78 \\
\hline & Fuel flow & 0.21 & 0.24 & 0.18 & 0.24 & 0.26 & 0.27 & 0.25 & 5.32 \\
\hline & Jackson 2.11 & 0.28 & 0.29 & 0.20 & 0.66 & 0.32 & 0.34 & 0.31 & 0.09 \\
\hline & Wave Power & 0.52 & 1.12 & 7.24 & 2.42 & 0.92 & 4.74 & 0.94 & 0.07 \\
\hline & I. 6.20 & 0.56 & 0.23 & 0.25 & 0.43 & 1.79 & 0.77 & 3.86 & 0.21 \\
\hline & I.9.18 & 1.15 & 0.87 & 0.25 & 0.46 & 1.47 & 4.74 & 0.26 & 0.12 \\
\hline & I.15.3x & 0.35 & 0.16 & 0.15 & 0.28 & 0.36 & 0.19 & 0.15 & 0.18 \\
\hline & I.15.3t & 0.34 & 0.23 & 0.19 & 0.25 & 0.15 & 0.26 & 0.21 & 0.21 \\
\hline & I. 30.5 & 0.20 & 0.19 & 0.15 & 0.76 & 0.21 & 0.21 & 0.20 & 0.14 \\
\hline & I. 32.17 & 0.85 & 0.81 & 0.65 & 28.23 & 1.74 & 6.36 & 0.71 & 0.11 \\
\hline & I. 41.16 & 0.40 & 0.30 & 0.16 & 13.37 & 1.03 & 2.10 & 0.49 & 0.05 \\
\hline & I. 48.20 & 0.16 & 0.16 & 0.14 & 0.23 & 0.20 & 0.21 & 0.19 & 0.19 \\
\hline & II.6.15a & 0.58 & 0.33 & 0.30 & 20.72 & 1.03 & 1.30 & 0.61 & 0.00 \\
\hline & II.11.27 & 0.18 & 0.17 & 0.15 & 0.19 & 0.21 & 0.38 & 0.17 & 0.18 \\
\hline & II.11.28 & 0.30 & 0.29 & 0.24 & 0.38 & 0.36 & 0.35 & 0.33 & 0.35 \\
\hline & II. 35.21 & 2.21 & 0.62 & 0.55 & 0.55 & 3.22 & 4.52 & 2.37 & 0.20 \\
\hline & III.9.52 & 29.77 & 5.65 & 9.22 & 5.50 & 33.68 & 29.77 & 22.17 & 0.28 \\
\hline & III.10.19 & 0.49 & 0.21 & 0.32 & 0.17 & 0.68 & 0.48 & 0.45 & 0.16 \\
\hline
\end{tabular}



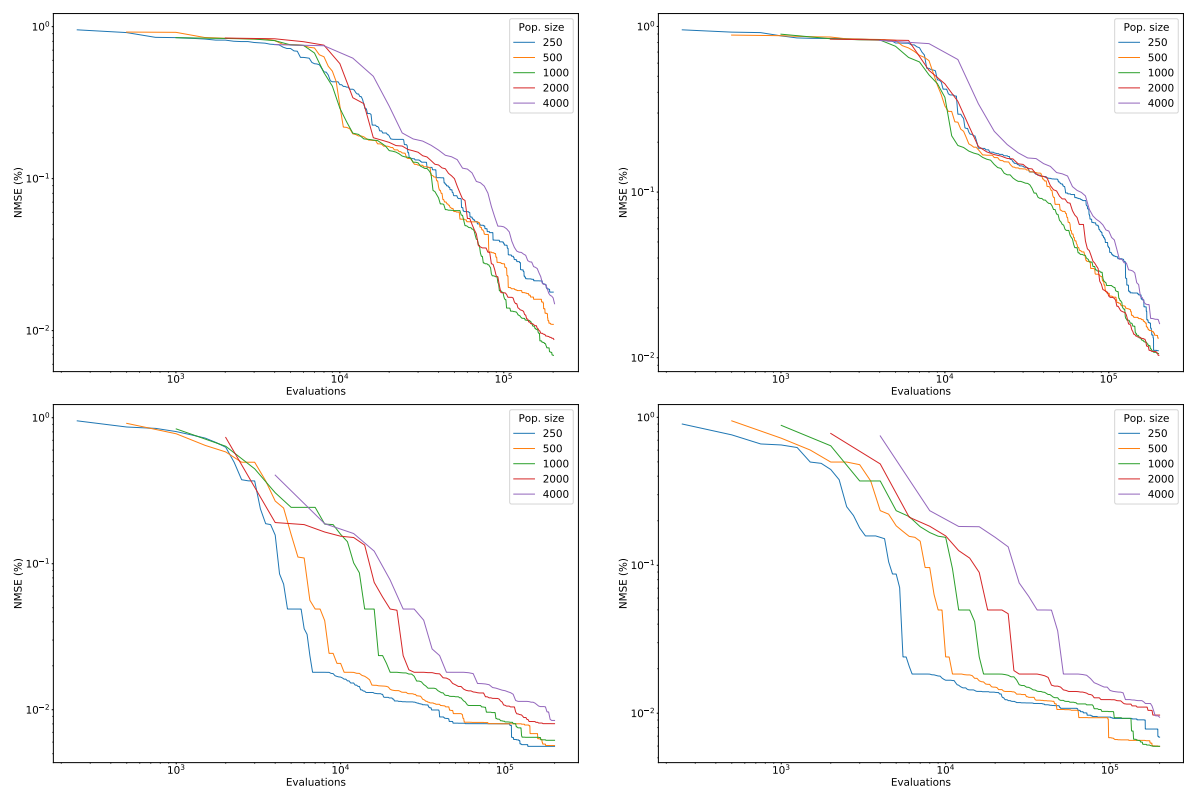

Figure 3: Convergence graphs of best quality over evaluations for different population sizes (top: GP, bottom: FI2POP, left: without noise, right with noise).

population size is potentially a sensitive parameter it is worthwhile to analyse how the algorithm convergence is influenced by different parameter settings. On the one hand smaller population sizes can improve the runtime on the other hand there is a danger that the population size becomes too small leading to loss of diversity and premature convergence.

For the experiment we have chosen one of the harder problem instances (Wave power) and have used the population sizes: 250, 500, 1000, 2000, and 4000. Figure 3 shows the best error over the number of evaluations (note: log-log scale) for GP and FI2POP with and without noise. It can be seen that comparable results can also be achieved with smaller population sizes. Overall, the population size of 1000 individuals, that we have originally used for the GP experiments, performs well compared to the other population sizes. The plots show no overly long plateaus in quality which would be indicative of premature convergence. 